\title{
Deconvolutive Clustering of Markov States
}

\author{
Ata Kabán and Xin Wang \\ School of Computer Science, The University of Birmingham, \\ Birmingham, B15 2TT, UK \\ $\{$ axk, xcw $\} @ c s . b h a m . a c . u k$
}

\begin{abstract}
In this paper we formulate the problem of grouping the states of a discrete Markov chain of arbitrary order simultaneously with deconvolving its transition probabilities. As the name indicates, this problem is related to deconvolutive blind signal separation. However, whilst the latter has been studied in the context of continuous signal processing, e.g. as a model of a real-room mixing of sound signals, our technique tries to model computer-mediated group-discussion participation from a discrete event-log sequence. In this context, convolution occurs due to various time-delay factors, such as the network transmission bandwidth or simply the typing speed of the participants. We derive a computationally efficient maximum likelihood estimation algorithm associated with our model, which exploits the sparsity of state transitions and scales linearly with the number of observed higher order transition patterns. Results obtained on a full day worth dynamic real-world Internet Relay Chat participation sequence demonstrate the advantages of our approach over state grouping alone, both in terms of penalised data likelihood and cluster clarity. Other potential applications of our model, viewed as a novel compact approximation of large Markov chains, are also discussed.
\end{abstract}

\section{Introduction}

The classical scenario that illustrates the problem of deconvolutive separation of mixed signals (deconvolutive blind source separation, DBSS) is as follows. Consider $K$ simultaneous speakers (sound signals) in a real room and $M$ microphone sensors. Each sensor receives a different convolutive mixture of the source signals. The mixing is convolutive due to the echo in a real room setting, i.e. several delayed and attenuated versions of the signals arrive at the sensor. The task is then to recover the individual speakers from the recorded mixture signals.

Due to its practical relevance in a number of problems, such as interference and echo cancellation, a large body of research on solving DBSS problems has been devised over the last decade. These include algebraic methods [12], predictability-based heuristics [18], information-theoretic methods [3] probabilistic and Bayesian methods [2] and various combinations thereof. A common technique is to work in the frequency domain, where the convolution becomes multiplication.

Our problem is conceptually related to DBSS, however it differs in a number of respects, which makes existing models and methods not directly applicable: 
(1) The observed signal is a symbolic sequence rather than a continuous realvalued stream. (2) The 'original source signals' that we assume to exist and try to recover are the cluster-membership probabilities of the symbols that make up the observed sequence. (3) There is a single observation channel. (4) The mixing proportions change over time. Further specificities and model assumptions will be discussed in more detail later.

An illustrative real-world example application, which is also in close analogy with the discussed classical DBSS problem setting is the modelling of a computer-mediated discussion session such as the Internet Relay Chat (IRC), as a dynamic social network. The observed signal in an IRC channel consists of typed contributions and the session is automatically logged in a sequential manner, as a single symbolic sequence. We make abstraction of the textual content here, instead, we focus on the participation sequence. Thus we have a symbolic sequence of user ID-s and what we are looking for is their cluster memberships, in order to identify user communities in this dynamic social network.

In this context, convolution occurs due to various time-delay factors, such as the network transmission bandwidth or simply the number and typing speed of the participants. In other words, we posit that it would be unrealistic to assume that consecutiveness of the contributions, as logged, is an accurate indicator of user interaction. Instead, our convolutive model allows for contributions situated at earlier time lags in the logged sequence to represent user links with a certain probability. The task is then to recover the most likely allocation of symbols to clusters, the mixing (interleaving) parameters and the convolution filter, i.e. the probability with which earlier time lags contribute towards the cluster structure.

There are many studies of community identification in static settings and with given network connections. $[10,13]$. However, dynamic analyses have been less researched and represent increasing interest recently $[6,15]$. To our best of knowledge, our deconvolutive clustering approach is new. However the method that we present is not restricted to this application but more generally applicable, in principle, to approximating large Markovian chains.

\section{The model}

Let $X=\left\{x_{1}, x_{2}, \cdots, x_{N}\right\}$ denote a symbolic sequence with each symbol $x_{n} \in$ $\{1,2, \cdots, T\}$ in a $T$-symbol state space. We assume that $X$ is a stationary $L$-th order Markov chain, i.e. $P\left(x_{n} \mid x_{n-1}, \cdots, x_{1}\right)=P\left(x_{n} \mid x_{n-1}, \cdots, x_{n-L}\right)$. We then define a compact approximation to the full $L$-th order transition probability at any discrete time $n$ as the following convolutive mixture.

$$
P\left(x_{n} \mid x_{n-1}, \cdots, x_{n-L}\right)=\sum_{l=1}^{L} e_{l} \sum_{k=1}^{K} s_{x_{n}, k} a_{k, x_{n-l}}
$$

The parameters of this model will be sought in the form of probabilities, as follows. The quantities of primary interest are sought as cluster membership probabilities $a_{k, s} \equiv P(k \mid s)$ so for all symbols $s$, we impose $\sum_{k} a_{k, s}=1, \forall s$. 
Likewise, the convolution filters $e_{l} \equiv P(l)$ will signify the probability with which each time lag, up to a memory depth of $L$, contributes towards the state clustering. Further, in order to obtain the required transition probability from this decomposition, it is convenient to also seek for the remaining mixing parameter in a probabilistic form, i.e. $s_{x_{n}, k} \equiv P\left(x_{n} \mid k\right)$.

\section{$2.1 \quad$ Interpretations}

Since all parameters of our model (1) are probabilities, we can interpret $k$ as the outcome of a latent class variable $\Xi \in\{1,2, \ldots, k, \ldots, K\}$. Likewise, we can also interpret $l$ as the outcome of another discrete latent variable $\Lambda \in\{1,2, \ldots, l, \ldots, L\}$ - the time delay variable. Then the complete data likelihood can be written as the following.

$$
\mathcal{L}^{C}=\prod_{n} \prod_{l} \prod_{k} P(l)^{\delta\left(\Lambda_{n}=l\right)}\left\{P\left(x_{n} \mid k\right) P\left(k \mid x_{n-l}\right)\right\}^{\delta\left(\Xi_{n}=k, \Lambda_{n}=l\right)}
$$

where $\delta$ is the Kronecker delta function. So at each discrete event we have a latent time delay and a latent class. The observed $L$-th order transition probability is assumed by this model to be generated by instantiating these latent variables.

The expectation of the log of (2) is the following.

$E\left[\log \mathcal{L}^{C}\right]=\sum_{n, l, k} P\left(\Xi=k, \Lambda=l \mid x_{n}, x_{n-1}, \ldots, x_{n-L}\right) \log \left\{P(l) P\left(x_{n} \mid k\right) P\left(k \mid x_{n-l}\right)\right\}$

This may then be maximised employing the Expectation Maximisation [8] methodology in order to estimate the model parameters. Doing so is straightforward, however tedious for this model, since the posterior probabilities of the discrete latent variables would need to be computed and stored for every possible L-gram. Therefore for deriving an efficient estimation algorithm, the form (1) will be manipulated so that the posterior probabilities need not be computed explicitly during the parameter estimation. The latent discrete variable view is however useful for the interpretation of the model and the posteriors may be computed for inference purposes after the parameter estimation is complete.

\subsection{Relation to existing discrete sequence models}

State aggregation in first order Markov chains through a discrete 'bottleneck' latent variable has been studied before and it is known as the aggregate Markov model [17]. This was previously used e.g. in language modelling $[17,11]$ and bibliometric analysis [7]. The aggregate Markov model seeks for the first order transition probabilities in the following form.

$$
P\left(x_{n} \mid x_{n-1}\right)=\sum_{k=1}^{K} P\left(x_{n} \mid k\right) P\left(k \mid x_{n-1}\right)
$$

However, extensions to higher order models have not been considered previously. 
A model that could be related to the problem of deconvolving transition probabilities, in the manner we employ it, was studied in statistics and it is known as the mixed transition model $[4,1,14]$. This has the following form:

$$
P\left(x_{n} \mid x_{n-1}, \cdots, x_{n-L}\right)=\sum_{l=1}^{L} P(l) P\left(x_{n} \mid x_{n-l}\right)
$$

A slightly different version, having separate transition matrices for all lags appears as the mixed memory Markov model [16]. In the mentioned works the goal has been to approximate a higher order Markov chain by a model that uses fewer parameters. The goodness of approximation has been studied [14,4]; moreover, the steady-state distribution was shown to be given by the principal eigenvector of the stochastic matrix in (4), $P\left(s \mid s^{\prime}\right)$ [1] where $s, s^{\prime}$ are the symbols in the dictionary.

We can view our model (1) as a combination of an aggregate Markov model and a mixed transition Markov model. Although our primary motivation in this paper has been to model the convolutive mixing process, owing to the results of $[14,4,1]$, it also follows that our model (1) provides an approximate solution to clustering the states of a higher order Markov chain.

Finally, it should be pointed out that despite we make use of hidden variables (as discussed in Sec.2.1), our model is different from the hidden Markov model (HMM) [19]. This is evident from the fact that we are using an approximation of an $L$-th order Markov model [4] of the observed sequence, whereas the HMM makes no Markov assumption of any order on the observed sequence. Instead, it makes the (first order) Markov assumption on a hidden sequence. The difference has several implications. From the point of view of parameter interpretation, we can regard $P(l)$ as the integrated saliency of past information at progressive temporal lags. These provide explicit credit-assignments for each time lag up to the depth $L$ of the Markov process, which may serve e.g. as a compact characterisation of the sequence ${ }^{1}$ There is no such credit-assignment indicator in HMMs. Finally, from the computation complexity point of view, the estimation of a HMM requires time quadratic in the number of hidden states, whereas, as we will see in the next section, our method can be estimated in time that is linear with the number of non-zero observed higher-order transition patterns. In consequence we view our model as being complementary to HMM both technically and in terms of functionality.

\subsection{Maximum likelihood estimation for deconvolutive state clustering}

In this section we derive an efficient iterative estimation algorithm for the deconvolutive clustering of Markov states, based on maximum likelihood (ML). Simple

\footnotetext{
${ }^{1}$ Although outside the scope of this paper, we experimentally found that the distribution $P(l)$ obtained from sequences that represent computer-mediated discussions tends to have high entropy, while those that represent individual activity have low entropy.
} 
manipulations of (1) yield the log likelihood of a sequence $X=\left\{x_{1}, \cdots, x_{N}\right\}$ under the model as follows:

$$
\log P(X \mid \Theta)=\sum_{t_{0}, t_{1}, \cdots, t_{L}=1}^{T} N_{t_{0}, t_{1}, \cdots, t_{L}} \log \sum_{l=1}^{L} e_{l} \sum_{k=1}^{K} a_{k, t_{l}} s_{t_{0}, k}
$$

where $\left(t_{0}, t_{1}, \cdots, t_{l}, \cdots, t_{L}\right)$ is used to denote an $(L+1)$-gram, such that $\left(x_{n}=\right.$ $\left.t_{0}, x_{n-1}=t_{1}, \cdots, x_{n-L}=t_{L}\right)$, for some $n$, where $t_{0}, t_{1}, \cdots, t_{l}, \cdots, t_{L}$ are symbols $\in\{1,2, \cdots, T\}$ in a dictionary of size $T$. Further, $N_{t_{0}, t_{1}, \cdots, t_{L}}$ denotes the frequency of the $(L+1)$-gram specified in the index, i.e. the frequency of the subsequence $t_{L} \rightarrow t_{L-1} \rightarrow \cdots \rightarrow t_{0}$. Finally, as earlier, $l \in\{1, \cdots, L\}$ stands for time-lags.

Maximising (5) with respect to all the parameters $e_{l}, a_{k, t_{l}}$ and $s_{t_{0}, k}$ under the requirement of being probabilities, is carried out iteratively, by alternating optimisation. This yields the following multiplicative fixed point updates:

$$
\begin{aligned}
& e_{l}^{i+1} \propto e_{l}^{i} \sum_{t_{0}, t_{1}, \cdots, t_{L}=1}^{T} \sum_{k=1}^{K} \frac{N_{t_{0}, t_{1}, \cdots, t_{L}} a_{k, t_{l}}^{i} s_{t_{0}, k}^{i}}{\sum_{l^{\prime}} e_{l^{\prime}}^{i} \sum_{k^{\prime}} a_{k^{\prime}, t_{l^{\prime}}}^{i} s_{t_{0}, k^{\prime}}^{i}} \\
& a_{k, t_{l}}^{i+1} \propto a_{k, t_{l}}^{i} \sum_{t_{0}, \cdots, t_{l-1}}^{T} \cdots \sum_{t_{l+1}, \cdots, t_{L}=1}^{T} \frac{N_{t_{0}, t_{1}, \cdots, t_{L}} e_{l}^{i} s_{t_{0}, k}^{i}}{\sum_{l^{\prime}} e_{l^{\prime}}^{i} \sum_{k^{\prime}} a_{k^{\prime}, t_{l^{\prime}}}^{i} s_{t_{0}, k^{\prime}}^{i}} \\
& s_{t_{0}, k}^{i+1} \propto s_{t_{0}, k}^{i} \sum_{t_{1}, \cdots, t_{L}=1}^{T} \sum_{l=1}^{L} \frac{N_{t_{0}, t_{1}, \cdots, t_{L}} e_{l}^{i} a_{k, t_{l}}^{i}}{\sum_{l^{\prime}} e_{l^{\prime}}^{i} \sum_{k^{\prime}} a_{k^{\prime}, t_{l^{\prime}}}^{i} s_{t_{0}, k^{\prime}}^{i}}
\end{aligned}
$$

where $\propto$ stands for proportionality and $i$ is the iteration index. The iterated application of the above updates is guaranteed to converge to a local optimum of the likelihood. This is because each of the above updates can also be derived as the consecutive application of a complete E-step and an M-step for one parameter set only, while keeping the remaining parameters fixed at their current values (cf. the interpretation discussed in Sec. 2.1.).

\subsection{Scaling}

The worst-case time complexity per iteration of our algorithm is $O\left(T^{L+2} \times L \times\right.$ $K)$. However, typically, real data tend to be sparse and this is what our algorithm exploits. Indeed, it can easily be seen from the updates, that whenever a count $N_{t_{0}, t_{1}, \cdots, t_{L}}$ is zero, the fraction is also zero and therefore needs not be evaluated. Let $S$ denote the number of non-zero observed $(\mathrm{L}+1)$-gram counts. Expressed in terms of $S$, our algorithm scales as $O(S \times L \times K)$. Typically $L \times K \ll S$, so the scaling is linear with the number of observed non-zero $(L+1)$-gram counts.

\subsection{Posterior computations}

As already pointed out, computing the posterior probabilities of the discrete variables is not required for estimating the parameters. Instead, once the parameter estimation is complete, we may wish compute the discrete posteriors 
once, in order to make further inferences about the data. This is in contrast with existing solutions of related models such as the aggregate Markov [17,11] and the mixed transition Markov model $[14,17]$, and could be applied to those as well.

Given the estimated parameter values, the joint posterior can be written as the following.

$$
P\left(k, l \mid t_{0}, t_{1}, \cdots, t_{L}\right)=P\left(l \mid t_{0}, t_{1}, \cdots, t_{L}\right) P\left(k \mid l, t_{0}, t_{1}, \cdots, t_{L}\right)
$$

Then, for each time-window of size $(\mathrm{L}+1)$, we obtain the posterior class $P\left(k \mid t_{0}, t_{1}, \cdots, t_{L}\right)=\sum_{l} P\left(k, l \mid t_{0}, t_{1}, \cdots, t_{L}\right)$ by marginalisation.

The two terms in (6) are computed using the model parameters and applying Bayes' theorem:

$$
\begin{gathered}
P\left(k \mid l, t_{0}, t_{1}, \cdots, t_{L}\right) \propto a_{k, t_{l}} s_{t_{0}, k} \\
P\left(l \mid t_{0}, t_{1}, \cdots, t_{L}\right) \propto N_{t_{0}, t_{1}, \cdots, t_{L}} e_{l} \sum_{k} a_{k, t_{l}} s_{t_{0}, k}
\end{gathered}
$$

The latter, $P\left(l \mid t_{0}, t_{1}, \cdots, t_{L}\right)$, gives the posterior distribution of time delays in each $(\mathrm{L}+1)$-window, and naturally, its maximum argument provides the most probable posterior delay in a particular window. So it can be used to reconstruct the a-posteriori transition graph, or, in other words, the inferred links between the states.

\subsection{Clustering the states}

In order to obtain cluster labels for the states (symbols), based on the model, we may proceed in several alternative ways.

One option is to use the parameters $a_{k, t_{l}}$, which, by the model design (cf. Sec.2), sum to one w.r.t. $k$, for each symbol $t_{l}$ from the dictionary and are meant to be interpretable as cluster memberships. It is useful to observe that during the iterative maximum likelihood estimation algorithm, each step minimises a weighted sum of entropies of all parameters [21]. Therefore if there are clusters in the sense defined by the model, the entropy of $a_{., t_{l}} \equiv P\left(. \mid t_{l}\right)$ may reach a reasonably low value at convergence, so that it is sensible indeed to interpret $\operatorname{argmax}_{k} a_{k, t_{l}}$ as the cluster label of symbol $t_{l}$. This can then be used to group similar states together. This procedure is convenient, since the parameters $a_{., t_{l}}$ are readily available and so only the inferred links need to be computed after completing the parameter estimation.

A second procedure for labelling the states falls out naturally from the posterior computations. This is to consider the context-conditional cluster-label $\operatorname{argmax} P\left(k \mid t_{0}, t_{1}, \cdots, t_{L}\right)$ as the label of $t_{0}$ conditional on the previous L-gram. $k$

Thirdly, we may obtain unconditional labels for the states by applying Bayes' rule and integrating over the contexts in the prior:

$$
P\left(k \mid t_{0}\right) \propto P(k) P\left(t_{0} \mid k\right) ; \text { where } P(k) \propto \sum_{t_{0}, t_{1}, \cdots, t_{L}=1}^{T} N_{t_{0}, t_{1}, \cdots, t_{L}} P\left(k \mid t_{0}, t_{1}, \cdots, t_{L}\right)
$$

and where $P\left(t_{0} \mid k\right) \equiv s_{t_{0}, k}$ is available. 


\section{Finding communities in a dynamical social network}

Here we apply our deconvolutive state clustering method to the problem of community identification from dynamic social networks.

Social network analysis $[10,13]$ is an interdisciplinary area dealing with the study of the structure of human relationships and associations which take the form of a network. Although most of these relations are dynamic in their nature, existing approaches to social network analysis, with very few exceptions [15], typically neglect the time component. Social networks are typically represented in the form of a graph with observable connections, and the analysis proceeds using various graph partitioning techniques.

However, we argue that the time component should be taken more seriously in many cases, and our proposed method provides a viable approach for doing so. In order to demonstrate this, we carry out (1) a data-driven evaluation of whether or not our proposed temporal deconvolution helps to better explain/model the dynamic social network, and (2) whether or not it helps obtaining clearer community structures.

The data that we use here is a sequence formed by real-world dynamic chat participation, collected from Internet Relay Chat (IRC) lines [5]. It consists of a stream of about one day worth discussions, totalling $N=25,355$ contributions from $T=844$ different chat participants. We study the symbolic sequence of user ID-s, making abstraction of the textual content of the contributions. The latter has been the focus of previous study [5], while here we are looking for communities (as state clusters) based on user interactions rather than based on topical similarities. The temporal connections in such data are quite sparse - the number of observed $(L+1)$-grams $(L \in\{1 \cdots 10\})$ is 14030, 23808, 24790, 24968, 25040, 25085, 25128, 25163, 25193, 25216 respectively, far less than $844^{L+1}$. Owing to the ability of our algorithm to take advantage of data sparseness to reduce computations, as discussed in Section 2.4, running our algorithm on this data takes less than a minute on a standard computer.

\subsection{Penalised data likelihood and selecting the best model order}

For each combination of $K$ and $L$, the models are trained 20 times to avoid local optima. The Akaike Information Criterion (AIC) is used to determine the optimal model order. Since we estimate our models by Maximum Likelihood, and our primary aim is data explanation, this criterion is appropriate [20]. The AIC has the simple form of a penalised data likelihood:

$$
A I C=-2 \log P(X \mid \Theta)+2 P
$$

where $P$ is the number of free parameters in the model, which in our case is $(L-1)+(T-1) K+(K-1) T$. The optimal model order is then given by the $\mathrm{L}$ and $\mathrm{K}$ pair that maximises $\mathrm{AIC}$.

Fig. 1 shows the AIC curves of our models. As we can see, all deconvolutive models (i.e. $L>1$ ) achieve higher scores than the Aggregate Markov (i.e. 


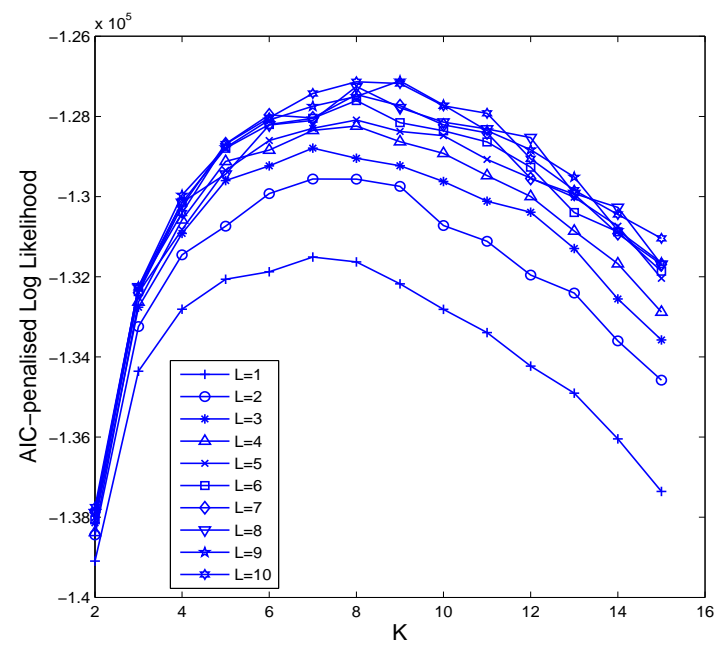

Fig. 1. The AIC curves (AIC values against number of clusters) for various models with differing order $L$. The model at $L=1$ reduces to the aggregate Markov (AM) model. Clearly, all models with $L>1$ outperform the AM and the best model order is found to be at $L=9, K=9$. Thus, the optimum number of clusters in this data is 9 , with a memory depth of 9 past symbols.

$\mathrm{L}=1$ ), which tries to group the states without a deconvolution model, based on consecutive (first-order) relationships. This demonstrates the convolutive models contribute to increasing the data likelihood under the model sufficiently to justify the extra parameters. In consequence, we have a data-driven evidence that our deconvolutive clustering model is more appropriate for modelling the dynamic social network analysed, in comparison to state aggregation alone.

\subsection{Cluster clarity}

Next, with the optimal model order selected above $(L=9, K=9)$, we investigate how does our deconvolutive model compare with state aggregation alone, in terms of the clarity of the clusters found. To this end, we make use of the posterior computations as described in Section 2.5. We take the maximum argument of the posterior time delay in each consecutive window, $l^{*}=$ $\operatorname{argmax} P\left(l \mid t_{0}, t_{1}, \cdots, t_{l}, \cdots, t_{L}\right)$, and use that to infer the de-convolved posterior transition matrix. Denoting this matrix by $\boldsymbol{M}$, each $M(i, j)$ will contain the frequency of symbol $j$ having triggered symbol $i$, after some time delay. $M$ is easily constructed by scanning the sequence and increasing $M\left(t_{0}, t_{l^{*}}\right)$ by 1 for each symbol. Further, we use any of the state cluster labelling procedures, as described in Section 2.6, to reorder M by grouping the states that are assigned the same label. 
Fig.2. shows the raw data in the form of a first-order adjacency graph, having the states in alphabetical order of the user IDs (upper left plot), the same graph with states reordered according to cluster labels obtained from the best Aggregate Markov model (upper right plot), and the results of the optimal deconvolutive clustering model (i.e. $\mathrm{L}=9, \mathrm{~K}=9$, cf. Fig.1.): The inferred posterior de-convolved adjacency matrix $(\boldsymbol{M})$, reordered using labels obtained from parameters of the model, i.e. $\underset{k}{\operatorname{argmax}} a_{k, t_{l}}$ is shown on the lower left plot and $\boldsymbol{M}$ reordered using the context-conditional labels, i.e. $\operatorname{argmax} P\left(k \mid t_{0}, t_{1}, \cdots, t_{l}, \cdots, t_{L}\right)$ is on the lower right plot. It is visually most apparent from these plots, that even though state aggregation alone does display some structure, a much clearer clustering has been obtained from our deconvolutive approach. Another observation is that the two lower plots are qualitatively similar. Using the marginal cluster labels, argmax $P\left(k \mid t_{0}\right)$ (omitted) has been qualitatively similar as well.

In order to provide a quantitative comparison in terms of cluster clarity, we use classical numeric measures to express the goodness of clustering: the strength of intra-connectivity vs. inter-connectivity in the identified clusters of states. We define the intra-connectivity $\mathcal{C}_{k i}$ of a cluster $k$ to be the density of links between members of that cluster, while inter-connectivity $\mathcal{C}_{k o}$ will refer to the density of links between members of different clusters. More formally,

$$
\mathcal{C}_{k i}=\frac{1}{\left|T_{k}\right| \times\left|T_{k}\right|} \sum_{i, j \in T_{k}} n_{i j} ; \quad \mathcal{C}_{k o}=\sum_{l=1, l \neq k}^{K} \frac{1}{\left|T_{k}\right| \times\left|T_{l}\right|} \sum_{i \in T_{k}} \sum_{j \in T_{l}}\left(n_{i j}+n_{j i}\right)
$$

where, $T_{k}, T_{l}$ denotes the state space of cluster $k$ and $l ; n_{i j}$ and $n_{j i}$ are transition counts in a reordered transition matrix. Table 1 summarises the results comparatively, for the optimal deconvolutive clustering (DC) model ( $\mathrm{K}=9, \mathrm{~L}=9)$, using the three labelling schemes (cf. Sec. 2.6.) and the optimal Aggregate Markov Model. We also included HMM (with 12 states, selected using AIC) in this comparison, for completeness. All three labelling schemes of DC produce clearer clusters than AM and HMM, the overall winner being the labelling based on the class posteriors conditioned on the previous L-gram.

\subsection{Scalability}

Since the application demonstrated in the previous subsections is focused on a particular data set, now we assess the computation time requirements of our parameter estimation algorithm on data sets of increasing size. Figure 3 shows the required $\mathrm{CPU}$ time per iteration (on a $2 \mathrm{GHz}$ Intel processor), as a function of the number of nonzero entries. As expected, the scaling is indeed linear and this backs up the theoretical complexity given earlier in Section 2.4.

\section{Conclusions}

We formulated the problem of deconvolutive clustering and developed a probabilistic model for this problem. We discussed analogies with deconvolutive source 

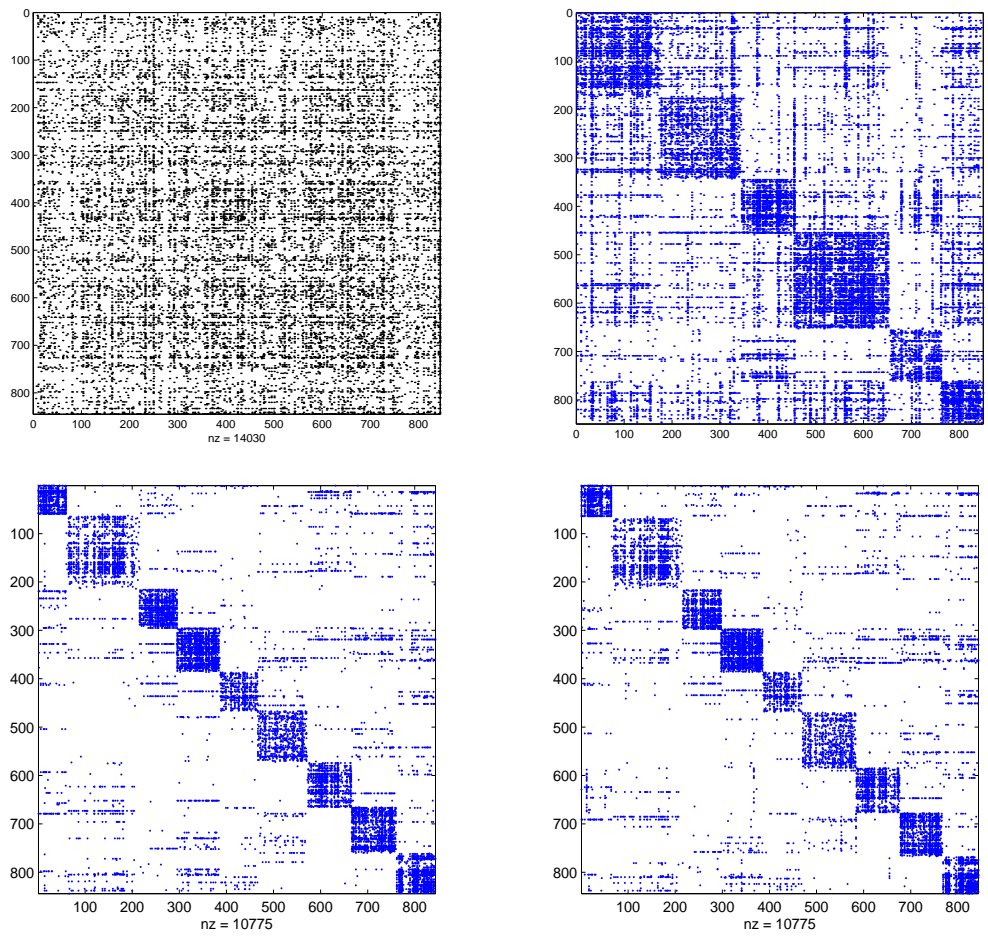

Fig. 2. The raw data shown as the first-order adjacency graph (upper left), the same graph with reordered states by using the best Aggregate Markov model (upper right), the inferred posterior de-convolved adjacency matrix $\boldsymbol{M}$, reordered using $\operatorname{argmax}_{k} a_{k, t_{l}}$ as obtained from the best $(\mathrm{K}=9, \mathrm{~L}=9)$ deconvolutive clustering model (lower left) and $M$ reordered using the context-conditional labels $\operatorname{argmax}_{k} P\left(k \mid t_{0}, t_{1}, \cdots, t_{l}, \cdots, t_{L}\right)$. The cluster clarity is superior for the deconvolutive models.

separation - which is fairly well-studied in signal processing - as well as similarities and differences with existing discrete sequence models. We derived a computationally efficient maximum likelihood estimation algorithm associated with our model, which exploits the sparsity of state transitions and scales linearly with the number of observed higher order transition patterns. Results obtained on a real-world dynamic social network demonstrated the advantages our approach over state grouping alone, both in terms of penalised data likelihood and cluster clarity.

Since our model can be viewed as a mixed-transition generalisation of the aggregate Markov model, and due to earlier results in statistics regarding the mixed transition model with shared transition probabilities as an approximation of a full higher order Markov chain [14], it follows that our approach is interpretable as providing an approximate solution to the problem of partitioning the 
Table 1. Quantitative comparison of cluster clarity. 'DC' = the optimal deconvolutive clustering model $(\mathrm{L}=9, \mathrm{~K}=9)$; AM: Aggregate Markov; HMM: Hidden Markov Model. $\mathcal{C}_{i}$ : intra-connectivity, averaged over all clusters (higher is better); $\mathcal{C}_{o}$ : intra-cluster connectivity (lower is better). The three variants of DC refer to the three different state labelling schemes (see text). All three labelling schemes of DC produce clearer clusters than AM, and even HMM, the overall winner being the labelling based on the class posteriors conditioned on the previous L-gram.

\begin{tabular}{c|c|c|c} 
Model & $\mathcal{C}_{i}$ & $\mathcal{C}_{0}$ & $\mathcal{C}_{i} / \mathcal{C}_{0}$ \\
\hline DC-context & $\mathbf{0 . 3 1 8 7}$ & $\mathbf{0 . 0 0 6 8}$ & $\mathbf{4 7 . 0 5 4}$ \\
DC-marginal & 0.3173 & $\mathbf{0 . 0 0 6 8}$ & 46.6618 \\
DC-parameter & 0.3171 & 0.0073 & 43.677 \\
AM & 0.13 & 0.0165 & 7.8788 \\
HMM $(\mathrm{K}=12)$ & 0.2616 & 0.0257 & 10.1962
\end{tabular}

states of a higher order Markov chain. Due to this, our algorithm may have further applications that are worthy of investigation, for example, in computing the steady state distribution of very large Markov models, such as those of interest in performance engineering and distributed systems [9].

\section{References}

1. S.R Adke and S.R Deshmukh. Limit Distribution of a Higher Order Markov Chain. Journal of the Royal Statistical Society B, 1988, 105-108.

2. H Attias and C.E Schreiner. Blind Source Separation and Deconvolution: The Dynamic Component Analysis. Neural Computation, 10(6): 1373-1424, 1998.

3. A.J Bell and T Sejnowski. An information Maximisation Approach to Blind Separation and Blind Deconvolution. Neural Computation, 7:1129-1159, 1995.

4. A Berchtold and A.E Raftery. The Mixture Transition Distribution Model for High-Order Markov Chains and Non-Gaussian Time Series. Statistical Science, 17(3):328-356, 2002.

5. E Bingham, A Kaban and M Girolami. Topic Identification in Dynamical Text by Complexity Pursuit, Neural Processing Letters 17: 1-15, 2003, pp. 69-83.

6. C Cortes, D Pregibon and C Volinsky. Communities of interest. Lecture Notes in Computer Science 2189. 2001.

7. D. Cohn and H. Chang. Learning to Probabilistically Identify Authoritative Documents. Proc. of 17th Int. Conf. on Machine Learning, pp. 167-174, 2000.

8. A.P Dempster, N.M Laird and D.B Rubin. Maximum Likelihood from Incomplete Data via the EM Algorithm. Journal of the Royal Statistical Society. Series B (Methodological), Vol. 39, No. 1. (1977), pp. 1-38.

9. N.J Dingle, P.G Harrison and W.J Knottenbelt. Uniformization and Hypergraph Partitioning for the Distributed Computation of Response Time Densities in Very Large Markov Models. Journal of Parallel and Distributed Computing, 64, 2004, pp. 908-920.

10. L Danon, A Diaz-Guilera, J Duch and A Arenas. Comparing Community Structure Identifications. Journal of Statistical Mechanics, 2005. P09008. 


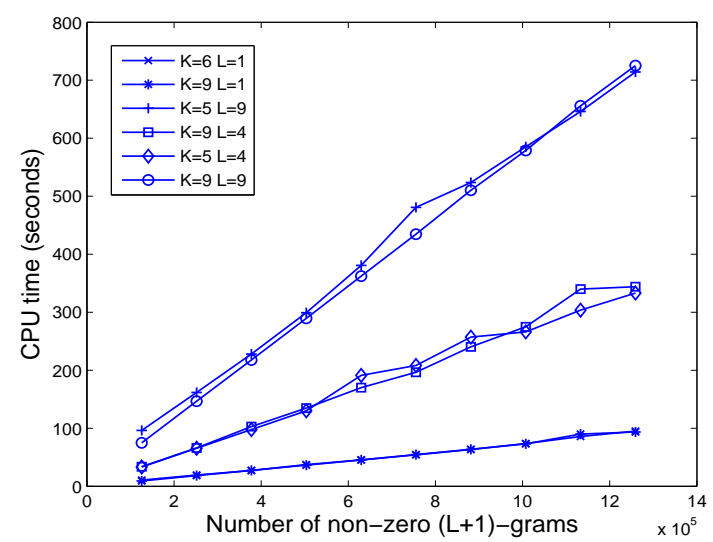

Fig. 3. CPU time versus the number of non-zero entries in the data, when the size of data is progressively increased.

11. D Gildea, T Hofmann. Topic-Based Language Models Using EM. Proc. of the 6th European Conference on Speech Communication and Technology, pp 2167-2170, 1999.

12. R.H Lambert. Multi-channel blind deconvolution: FIR matrix algebra and separation of multipath mixtures, Ph.D. Thesis, Univ. of Southern California, 1996.

13. M.E.J Newman. Detecting community structure in networks. Euro. Phys. Journal B, 38: 321-330, 2004.

14. A.E Raftery. A Model for High-order Markov Chains. Journal of the Royal Statistical Society, series B, 47:528-539, 1985.

15. P Sarkar and A Moore. Dynamic Social Network Analysis using Latent Space Models. Proc. Neural Information Processing Systems, 2005.

16. L.K Saul and M.I Jordan. Mixed Memory Markov Models: Decomposing Complex Stochastic Processes as Mixtures of Simpler Ones. Machine Learning, 37(1):75-87, 1999.

17. L. Saul and F. Pereira. Aggregate and Mixed-Order Markov Models for Statistical Language Processing. Proc. of the Second Conference on Empirical Methods in Natural Language Processing, pp. 81-89, 1997.

18. J.V. Stone: Blind deconvolution using temporal predictability. Neurocomputing 49(1-4): 79-86 (2002)

19. L.R Rabiner. A Tutorial on Hidden Markov Models and Selected Applications in Speech Recognition. Proc. of the IEEE, 77(2): 257-286, 1989.

20. B. Ripley. Pattern Recognition in Neural Networks. Cambridge Univ. Press, 1996.

21. K Rose, E. Gurewitz and G.C Fox. Statistical Mechanics and Phase Transitions in Clustering. Phys. Rev. Letters, vol. 65, no. 8, pp.945-948. 